\title{
Transtorno de estresse pós-traumático e uso de drogas ilícitas em mulheres encarceradas no Rio de Janeiro
}

\author{
Posttraumatic stress disorder and illicit drug use among incarcerated women in Rio de Janeiro
}

\author{
Byanka Quitete ${ }^{1}$, Beatriz Paulino², Francine Hauck², Aline Silva de Aguiar-Nemer³, Vilma Aparecida da Silva-Fonseca ${ }^{4}$ \\ 1 Pós-Graduação em Ciências Médicas, Universidade Federal Fluminense (UFF), Niterói, RJ, Brasil. \\ 2 Faculdade de Medicina, UFF, Niterói, RJ, Brasil. \\ 3 Departamento de Nutrição, Instituto de Ciências Biológicas, Universidade Federal de Juiz de Fora (UFJF), Juiz de Fora, MG, Brasil. \\ ${ }_{4}^{4}$ Núcleo de Ciências Comportamentais e do Desenvolvimento (NCCD), Departamento de Farmacologia e Fisiologia, Instituto Biomédico, UFF, Niterói, RJ, Brasil.
}

Recebido: 23/8/2011 - Aceito: 31/10/2011

\begin{abstract}
Resumo
Contexto: O risco para o desenvolvimento de transtorno de estresse pós-traumático (TEPT) entre mulheres encarceradas pode estar associado ao consumo de drogas. Objetivos: Determinar a frequência de TEPT entre mulheres encarceradas e sua relação com o uso de drogas na prisão. As voluntárias foram também avaliadas para a determinação de ansiedade-traço, depressão, eventos estressores e características sociodemográficas e comparadas quanto à presença ou não do transtorno. Métodos: Estudo transversal com mulheres encarceradas em que foram utilizados para a avaliação de TEPT os critérios determinados pelo DSM-IV, o Inventário Christo para descrição de uso de drogas, o Inventário de Ansiedade Traço-Estado e o Inventário Beck para avaliação de sintomas de ansiedade e depressão, respectivamente. Resultados: Das 134 mulheres avaliadas, 40,3\% apresentaram TEPT. Houve maior uso diário de cocaína entre as positivas para o transtorno $(\mathrm{p}<0,01)$. O uso diário de drogas ilícitas na prisão foi descrito por $53 \%$ das entrevistadas. Todas as participantes TEPT-positivas apresentavam algum grau de depressão em comparação às TEPT-negativas $(\mathrm{p}<0,05)$. Conclusão: A alta prevalência de TEPT encontrada é comparável à de populações de risco. Apesar de estarem em regime prisional, é alto o uso de drogas ilícitas. O maior uso de cocaína entre as detentas que apresentaram TEPT indica preferência por drogas estimulantes em mulheres com o transtorno.
\end{abstract}

Quitete B, et al. / Rev Psiq Clín. 2012;39(2):43-7

Palavras-chave: Estresse pós-traumático, transtornos da ansiedade, abuso de drogas, prisões.

\begin{abstract}
Background: The risk of developing posttraumatic stress disorder (PTSD) among incarcerated women may be associated with drug use. Objectives: To establish the frequency of PTSD in a sample of incarcerated women and its relationship with drug use inside prison. Anxiety and depression, nature of stressors and sociodemographic data were evaluated and the sample of PTSD-positive women was compared with the one of PTSD-negative women. Methods: The study had a cross-sectional design. The PTSD was evaluated according to the DSM-IV criteria. Drug consumption was assessed with the Christo Inventory for drug consumption. Anxiety was evaluated with the Spielberger Inventory and depression with the Beck Inventory. Results: Among the 134 examined women, $40.3 \%$ showed PTSD. There was a significantly higher daily use of cocaine among women with a positive PTSD diagnosis ( $<<0.01$ ). Daily consumption of illicit drugs was reported by $53 \%$ of the assessed women in prison. Compared to PTSD-negative women, all participants diagnosed as PTSD-positive showed some degree of depression ( $\mathrm{p}<0.05$ ). Discussion: The high prevalence of PTSD among incarcerated women is comparable to the prevalence of high-risk populations described in other studies. In spite of being in prison, consumption of illicit drugs was high. The association of PTSD with an increased cocaine use suggests a preference to consume stimulant drugs among incarcerated women with PTSD.
\end{abstract}

Quitete B, et al. / Rev Psiq Clín. 2012;39(2):43-7

Keywords: Posttraumatic stress disorder, anxiety disorder, substance-related disorder, prison.

\section{Introdução}

O transtorno de estresse pós-traumático (TEPT) foi inicialmente descrito em veteranos e sobreviventes civis de guerras 1 , porém, atualmente, verifica-se a sua importância diante do aumento de incidentes violentos que afetam a população civil urbana².

Os sintomas de TEPT podem ocorrer após vivenciar, testemunhar ou ser confrontado com um ou mais eventos que envolveram mortes ou graves ferimentos, reais ou ameaçados, ou uma ameaça à integridade física, própria ou de outros. Para que o diagnóstico seja estabelecido, esse evento deve necessariamente ter sido uma experiência acompanhada de uma resposta emocional específica em que o indivíduo apresentou intenso medo, impotência ou horror ${ }^{3}$.

A manifestação do TEPT é descrita como uma tríade psicopatológica em que há o desenvolvimento de três dimensões de sintomas: o reexperimentar do evento traumático, a evitação de estímulos a ele associados e entorpecimento da responsividade geral (não presente antes do trauma) e, ainda, a presença persistente de sintomas de hiperestimulação autonômica ${ }^{3}$. Nem todos os expostos a eventos traumáticos apresentam TEPT, fato que evidencia o papel de fatores predisponentes para o desenvolvimento do distúrbio.

A prevalência de TEPT encontrada na literatura varia de $1,0 \%{ }^{4}$ a $7,8 \%{ }^{2}$. Porém, a relação entre exposição e desenvolvimento do transtorno é reafirmada em estudos que descrevem prevalências de até $58 \%$ em grupos expostos a altos índices de criminalidade 5 . O Brasil apresenta taxas de homicídio intencional entre as maiores do mundo $0^{6}$ o que evidencia a alta exposição da população a estressores. A literatura descreve alguns fatores de risco para TEPT: sexo feminino, exposição a situações traumáticas prévias, ter história pessoal e familiar de transtornos mentais, separações precoces, eventos traumáticos na infância ${ }^{7}$.

Estudo realizado com mulheres encarceradas do Recife identificou que $87 \%$ sofreram algum tipo de violência física ou sexual que influenciou no desenvolvimento de depressão e abuso de drogas ${ }^{8}$. 
A exposição a eventos traumáticos com consequências psicológicas foi também descrita em mulheres prisioneiras de outros países9.

Quando se considera o fator uso e abuso de drogas, inúmeros trabalhos relataram sua ocorrência comórbida ao transtorno de estresse pós-traumático ${ }^{10-12}$. Essa associação também foi encontrada em amostras de estudos realizados em prisões ${ }^{8,13}$. Chilcoat e Breslau ${ }^{11}$ descreveram um risco quatro a cinco vezes maior de abuso e dependência de drogas em indivíduos que já tiveram TEPT em relação aos que não apresentam histórico do transtorno. Paralelamente, Brady et al. ${ }^{10}$ descreveram uma prevalência de $36 \%$ a 50\% de TEPT entre pacientes que procuram tratamento para distúrbios de uso de substâncias psicoativas.

Neste estudo, esperava-se encontrar alta prevalência de TEPT entre as mulheres avaliadas por causa da exposição aos fatores de risco já apresentados, podendo haver associação desse distúrbio com o consumo de drogas. Para avaliação da hipótese descrita e considerando que a população feminina desse universo encontra-se potencialmente ausente nos estudos nacionais, o objetivo do presente trabalho foi estimar a prevalência do TEPT entre mulheres encarceradas em um presídio do Rio de Janeiro e a sua associação com o consumo de drogas psicoativas dentro da prisão. Dada a importância da sobreposição de abuso de drogas e transtornos de humor e ansiedade, avaliou-se também a presença de humor depressivo e ansiedade-traço por meio de escalas. A caracterização sociodemográfica da amostra foi realizada. Possíveis diferenças entre mulheres diagnosticadas com TEPT ou não foram avaliadas.

\section{Métodos}

Todos os procedimentos realizados durante a execução do presente estudo foram aprovados pelo Comitê de Ética em Pesquisa da Universidade Federal Fluminense (CAAE: 0027.0.258.000-07) e pelas autoridades responsáveis pelas prisioneiras.

O Sistema Penitenciário do Rio de Janeiro abriga mulheres distribuídas nas três unidades prisionais femininas existentes: Penitenciária Talavera Bruce, Presídio Nelson Hungria e Penitenciária Joaquim Ferreira de Souza. A unidade prisional avaliada foi o Presídio Nelson Hungria, situado no Complexo Penitenciário de Gericinó (Bangu, Zona Oeste do Rio de Janeiro), por ser a porta de entrada no sistema penal do Rio de Janeiro, por onde passam todas as mulheres que devem cumprir pena de prisão. No período da pesquisa havia 468 detentas no presídio já sentenciadas ou aguardando julgamento.

Utilizou-se como critério de inclusão o tempo de encarceramento mínimo de cinco meses e máximo de dois anos. Dada a prioridade em estabelecer o padrão de uso de drogas atual, portanto de mulheres na rotina do ambiente prisional, o estabelecimento de tempo mínimo de prisão visou excluir mulheres cujo padrão atual de uso de drogas pudesse corresponder com mais fidelidade ao período pré-encarceramento. A lembrança do consumo antes do encarceramento poderia interferir nas informações sobre o consumo atual. Já o objetivo para o estabelecimento de tempo máximo foi minimizar as chances de inclusão de mulheres que seriam transferidas ou terminariam o cumprimento de suas penas durante o período de coleta de dados.

A direção do hospital forneceu uma lista oficial com 140 possíveis participantes que preenchiam os critérios de inclusão estabelecidos para participar da pesquisa. A amostra foi, portanto, de conveniência e o estudo, transversal.

Por meio da listagem recebida, as presidiárias foram convocadas em grupos de 10 e foram reunidas na sala de aula do presídio, onde receberam as explicações e orientações sobre a pesquisa e o preenchimento dos instrumentos. Após os esclarecimentos, permaneceram em sala aquelas que concordaram com os procedimentos. As que se recusaram a responder aos questionários foram liberadas com os devidos agradecimentos. As mulheres que concordaram em participar assinaram o Termo de Consentimento Livre e Esclarecido. Em seguida, todas preencheram, individualmente, os instrumentos lidos pelos pesquisadores quando havia dificuldade de leitura pelas voluntárias.
Se não havia dificuldade de leitura, os pesquisadores apenas se certificavam de que os instrumentos haviam sido preenchidos, e a orientação quanto ao procedimento era de que, caso fossem detectadas lacunas, era preciso esclarecê-las.

\section{Equipe}

Os instrumentos foram aplicados sob a supervisão de uma médica psiquiatra (VASF), sempre com a presença de uma psicóloga (BQ) treinada pela supervisora e membro da equipe de saúde do presídio. Auxiliada por duas estudantes de medicina, a psicóloga esteve presente durante toda a coleta de dados e participou da proposta do trabalho e da escolha de instrumentos, uma vez que este estudo fez parte dos requisitos para seu mestrado. As estudantes de medicina foram treinadas fora da instituição, aplicando os instrumentos a colegas e funcionários do Instituto Biomédico da UFF e na primeira visita ao presídio estiveram sob a orientação da psicóloga e da supervisora, psiquiatra. Nas demais, foram sempre acompanhadas pela psicóloga.

\section{Instrumentos}

Além do questionário construído pelos pesquisadores para traçar o perfil sociodemográfico, as participantes também preencheram os seguintes questionários padronizados: Inventário de Drogas ${ }^{14,15}$, Avaliação de Eventos Traumáticos ${ }^{12}$, Inventário de Ansiedade-Traço ${ }^{16}$ e Escala de Depressão ${ }^{17}$. Os pesquisadores utilizaram os critérios diagnósticos do DSM-IV para o diagnóstico de TEPT.

1) Questionário Sociodemográfico: foi dividido em três seções (dados pessoais, dados sobre a infância e dados de saúde), totalizando 23 perguntas que abrangiam grau de escolaridade das participantes e seus genitores, crescimento, consumo de drogas por pais e cuidadores, uso de drogas por companheiros, estado civil, dados obstétricos (número de gestações e de filhos), profissão antes da prisão e motivo da pena.

2) Quantificação de Uso de Drogas Psicoativas: as drogas utilizadas até um mês antes da pesquisa, a frequência do uso, a idade de início do uso e a quantidade consumida foram relatadas com o preenchimento do Inventário Christo ${ }^{14,15}$.

3) Avaliação de Eventos Traumáticos: o encarceramento é potencialmente um evento traumático, especialmente quando ocorre com violência física ou emocional. $\mathrm{O}$ impacto desse evento no desencadeamento de TEPT foi verificado. Para a detecção de outros eventos e do impacto emocional sobre a voluntária, uma lista de eventos potencialmente estressores foi apresentada, com abertura para a menção de algum evento omitido que tenha sido significativo de acordo com os critérios diagnósticos. Da lista de eventos traumáticos, foram assinalados aqueles aos quais estiveram expostas em algum momento da vida. Dentre os eventos assinalados, deveriam descrever se consideravam algum como traumático e assinalar o mais traumático e idade em que o vivenciaram.

4) Critérios Diagnósticos para Transtorno de Estresse Pós-Traumático/DSM-IV:a referência para o presente estudo foram os critérios diagnósticos estabelecidos na $4^{\mathrm{a}}$ edição do Manual de Diagnóstico e Estatística dos Distúrbios Mentais ${ }^{3}$ da Associação Americana de Psiquiatria (DSM-IV) para TEPT, os quais estabelecem a exposição a um evento traumático como pré-requisito (Critério A1) para preencher os demais critérios, além da sua validade diagnóstica ${ }^{18}$. Para o diagnóstico, exige-se que a pessoa tenha vivenciado, testemunhado ou sido confrontada com um ou mais eventos que envolveram mortes ou graves ferimentos, reais ou ameaçados, ou uma ameaça à integridade física, própria ou de outros.

A resposta ao evento traumático inclui intenso medo, impotência ou horror (critério A2). Os sintomas são distribuídos de acordo com sua natureza em critérios B (revivência persistente do evento), C (esquiva persistente a estímulos associados ao evento) e D (excitação aumentada). É também indispensável que o quadro persista por mais de um mês (critério E) e que haja sofrimento ou prejuízo clinicamente significativo no funcionamento social, ocupacional 
ou em outras áreas importantes da vida do indivíduo (critério F). O diagnóstico de TEPT requer preenchimento dos critérios A1 e A2, mínimo de um critério $\mathrm{B}$, três critérios $\mathrm{C}$ e dois critérios $\mathrm{D}$ e preenchimento dos critérios E e F. Apenas as detentas que classificaram um dos eventos como traumático foram avaliadas em entrevista baseada nos critérios diagnósticos da Associação Americana de Psiquiatria (DSM-IV) para a presença ou não de transtorno de estresse pós-traumático. Essa entrevista teve por referência a Escala de Avaliação de PTSD Administrada pelo Clínico (Clinician Administered PTSD Scale - CAPS) versão traduzida por L. M. Ito e publicada por M. C. Roso (2000) ${ }^{19}$. A escala foi modificada e utilizada na avaliação até o item 17 , que encerra o critério D (sintomas persistentes de aumento da excitabilidade). Os critérios E (duração da perturbação) e F (sofrimento clinicamente significativo ou prejuízo no funcionamento social ou ocupacional ou em outras áreas importantes da vida do indivíduo) foram avaliados por pergunta direta (E) e por perguntas abertas direcionadas ao envolvimento com as atividades oferecidas na prisão $(\mathrm{F})$.

5) O Inventário de Ansiedade-Traço: o Inventário de Ansiedade Traço-Estado, de Spielberger ${ }^{16}$, fornecido pelo CEPA (Centro de Psicologia Aplicada, RJ) e validado para a população brasileira ${ }^{20}$, foi projetado para ser autoaplicável, sendo de realização individual ou em grupo. O inventário é constituído por dois instrumentos independentes - traço e estado de ansiedade -, os quais enfocam abordagens distintas de ansiedade. Neste estudo foi utilizada apenas a escala traço de ansiedade para todas as detentas. $\mathrm{O}$ traço se refere às diferenças individuais relativamente estáveis quanto à ansiedade e à diferença nas reações a situações percebidas como ameaçadoras.

Esse instrumento é constituído por 20 afirmativas e 4 opções para a resposta: 1 - quase nunca; 2 - às vezes; 3 - frequentemente; 4 - quase sempre. Quanto mais alta a pontuação, maior o nível de ansiedade: 20 a 40 pontos indica baixa ansiedade; 41 a 60 , média; e 61 a 80 , alta ansiedade.

6) Escala de Depressão: o Inventário Beck para Depressão ${ }^{17}$, publicado em português ${ }^{18}$, consiste de 21 itens compreendendo sintomas e comportamentos das esferas cognitiva, vegetativa e do humor. O inventário foi apresentado a todas as participantes, as quais avaliaram cada item em uma escala com quatro graus de intensidade ou severidade, podendo a pontuação variar de 0 a 63. Foram utilizados, para diferenciar os níveis de sintomatologia depressiva, os critérios adotados por Gorenstein e Andrade ${ }^{20}$, que admitem pontuação igual ou superior a 16 para detectar disforia e igual ou superior a 21 para categorizar indivíduos com depressão.

\section{Análise estatística}

Os dados do presente estudo foram analisados por meio do programa SPSS 15.0. Os resultados foram expressos em percentual (\%), média e desvio-padrão $( \pm)$. A estatística foi paramétrica sempre que as variáveis obedeceram aos critérios de normalidade, homocedasticidade e continuidade. Do contrário, foi utilizada análise não paramétrica. Para análise comparativa, as mulheres foram divididas em dois grupos: TEPT-positivo (TEPT+, portadoras de TEPT) e TEPT-negativo (TEPT-, não portadoras de TEPT). Utilizou-se o teste do qui-quadrado para comparação de proporções e o teste Exato de Fischer quando necessário. O nível de significância estabelecido foi de $5 \%$.

\section{Resultados}

Dentre as 140 mulheres incluídas na listagem fornecida pela unidade prisional e que preenchiam os critérios de inclusão, uma se recusou a participar e cinco não se encontravam disponíveis no momento da coleta dos dados. Ao todo, 134 mulheres participaram da pesquisa.

$\mathrm{Na}$ avaliação das características sociodemográficas das participantes, foi observado que a maioria das mulheres entrevistadas estava na faixa etária entre 18 e 29 anos $(49,3 \%, \mathrm{n}=66)$, com idade média de 31,5 $\pm 10,4$ anos. A religião evangélica foi a mais prevalente, relatada por $44,8 \%(\mathrm{n}=60)$ das entrevistadas. Foi alto o índice de baixa escolaridade (ensino fundamental incompleto) entre as entrevistadas $(57,4 \%)$. Os artigos mais frequentes pelos quais as detentas cumpriam pena foram os que envolvem tráfico de drogas (especificamente: 12 - tráfico de drogas; 14 - associação ao tráfico de drogas; 33 - tráfico internacional de drogas), correspondendo à prisão de $55,2 \%$ das entrevistadas. A presença exclusiva materna foi a mais frequentemente descrita entre as entrevistadas que relataram receber quaisquer visitas ( $n=23,32,9 \%$ das 70 que relataram visitas).

A maioria das mulheres possuía companheiro e filhos vivos $(74,6 \%, \mathrm{n}=100)$, estando esses sob os cuidados de familiares $(55,9 \%$, $\mathrm{n}=75)$. O número médio de filhos entre as detentas que declararam já ter engravidado foi de $2,2 \pm 1,6$.

A baixa escolaridade também se repetiu entre as referências maternas $(63,4 \%)$ e paternas $(53,7 \%)$, sendo notável a alta taxa de desconhecimento ou não declaração das escolaridades materna e paterna $(23,9 \%$ e $38,1 \%$, respectivamente) pelas participantes.

Ao mesmo tempo, o trabalho abaixo dos 17 anos apresentou-se frequente, sendo descrito por $40,3 \%$ das entrevistadas. No entanto, não foi encontrada diferença estatisticamente significativa entre os grupos TEPT+ e TEPT- para os dados sociodemográficos apresentados.

Todas as mulheres sofreram exposição a algum evento potencialmente traumático, posto que a prisão, principalmente se acompanhada da ameaça de violência sexual, foi detectada como evento estressor por outros autores ${ }^{21}$. Trinta e sete mulheres $(27,6 \%)$, no entanto, não consideravam nenhum evento em suas vidas como traumático. As demais $(\mathrm{n}=97,72,4 \%)$ consideraram ter passado por algum trauma e incluíram as que apresentavam TEPT $(\mathrm{n}=54$, $40,3 \%$ ) e as que não apresentavam TEPT no momento do estudo (n $=43,32,1 \%)$. A maioria das entrevistadas que relataram vivência de um evento traumático possuía entre 18 e 29 anos quando o evento ocorreu $(n=39,39 \%)$. Apenas 16 mulheres relataram ter experimentado evento traumático na infância (até 12 anos), o que não permitiu análise estatística por faixa etária. Entretanto, comparadas às que vivenciaram eventos traumáticos antes e depois dos 18 anos, não houve diferença significativa na prevalência de TEPT.

Quando em liberdade, o consumo de drogas ilícitas foi relatado por $61(45,5 \%)$ entrevistadas, sendo mais frequente entre as que apresentaram TEPT $(57,4 \%)$ do que entre as que não apresentaram $(37,5 \%)$. Dessas 61 detentas, $72,1 \%(n=44)$ relataram convívio com pais usuários de drogas. $\mathrm{O}$ tabaco foi a droga mais utilizada, relatada por 92 mulheres $(68,6 \%)$. Dentre as detentas que faziam uso diário de tabaco, a média de cigarros foi de 12,6 $\pm 5,5$ cigarros/dia. Entretanto, 71 mulheres $(53 \%)$ relataram uso diário de pelo menos um tipo de droga ilícita. A droga ilícita mais consumida na amostra total foi Cannabis $(n=56,41,7 \%)$, seguida de cocaína/crack $(n=37,27,6 \%)$. Houve diferença significativa quanto ao consumo de cocaína dentro da prisão: $42,5 \%$ das TEPT-positivas descreveram uso diário versus $17,5 \%$ das TEPT-negativas $(\mathrm{p}<0,01)$.

Resultados da avaliação de transtornos de ansiedade e depressão indicaram que, independentemente da presença ou não de TEPT, todas as mulheres entrevistadas que aceitaram responder aos questionários apresentaram algum grau de ansiedade. Por outro lado, todas as mulheres com TEPT preencheram critérios para algum grau de depressão, sendo o grau moderado o mais frequente entre elas $(64,8 \%)$. Diferença significativa foi encontrada quando os dois grupos (TEPT-positivo e TEPT-negativo) foram comparados $(\mathrm{p}<0,05)$.

\section{Discussão}

O consumo de drogas ilícitas dentro da prisão constatado no presente estudo foi alto (53\%) e está de acordo com os encontrados em prisões americanas, onde mais de $70 \%$ das mulheres encarceradas relatam história de uso de drogas nas prisões federais e mais de $80 \%$, nas prisões estaduais e locais ${ }^{22}$. Exames toxicológicos de urina, realizados no ano de 1999 em prisões americanas, mostraram testes positivos em $67 \%$ das mulheres ${ }^{22}$. O fato de o crime de tráfico de drogas ser o mais frequente entre as mulheres aprisionadas já foi descrito em estudos 
conduzidos no Sistema Penal do Rio de Janeiro ${ }^{23}$ e foi confirmado neste estudo. Apesar disso, a alta frequência de uso de drogas ilícitas, em especial maconha e cocaína, encontrada no presente trabalho é uma evidência da presença do tráfico dentro das prisões. Essa constatação é paradoxal, posto que o crime pelo qual foram condenadas faz parte do dia a dia do local onde cumprem pena.

As voluntárias do presente estudo referiram consumo de drogas ilícitas já alto antes do encarceramento $(45,5 \%)$, revelando também alto consumo dessas drogas e álcool por seus pais. O consumo de drogas pelo indivíduo tem início na própria família, o que origina um círculo vicioso de eventos desfavoráveis evidenciado no presente estudo pela perda da liberdade. Prevalência semelhante foi encontrada por Reed et al. ${ }^{8}$ entre as mães detentas do Recife. $O$ consumo de drogas psicoativas muitas vezes indica automedicação para desconforto psíquico, como a depressão, que é comorbidade comum em mulheres usuárias de drogas 24,25 . No presente estudo, o consumo diário de cocaína foi mais frequente entre as mulheres que apresentavam TEPT e estas apresentavam com maior frequência algum grau de depressão.

A prevalência de TEPT entre as participantes foi alta (40,3\%) e comparável às maiores taxas encontradas em populações de maior risco descritas na literatura ( $3 \%$ a $58 \%$ ), como os veteranos de guer$\mathrm{ra}^{1}$. Consta também na literatura que a causa mais comum de TEPT em mulheres é a violência sexual ou abuso físico quando crianças ${ }^{26}$. Também no presente estudo, o abuso sexual foi o evento mais relacionado ao TEPT, embora os dados não tenham sido analisados estatisticamente por causa do pequeno número de voluntárias em cada categoria. Falbo et al. ${ }^{27}$ demonstraram em detentas do Recife que o trauma de abuso sexual, físico ou psicológico sofrido na infância e na adolescência levou-as a perpetuarem esses abusos na vida adulta com seus filhos, podendo ser um marcador preditivo para transtornos de conduta e posterior comportamento criminoso ${ }^{27}$.

Apesar de todas as entrevistadas estarem encarceradas, o que em si poderia ser um evento traumático, já que devido às condições de vida dentro dos presídios a integridade física é ameaçada de fato ou constantemente imaginada, a prisão não foi assim considerada por $27,6 \%$ das detentas. Embora a relação entre vulnerabilidade individual e desenvolvimento de transtornos de ansiedade já seja conhecida $^{28}$, pouco é encontrado a respeito dos fatores determinantes ou influentes nessa vulnerabilidade. $\mathrm{O}$ que faz um indivíduo considerar a prisão - ou qualquer outro evento - como traumático abre espaço para outro questionamento: situações não consideradas ou listadas como traumáticas para a maioria da população poderiam ser excepcionalmente desencadeadoras de TEPT em indivíduos suscetíveis?

Em seu grande levantamento epidemiológico realizado nos Estados Unidos, Kessler et al. ${ }^{2}$ identificaram que, apesar de mais homens (61\%) que mulheres (51\%) terem passado por eventos traumáticos em suas vidas, a ocorrência de TEPT era duas vezes maior na população feminina (10\%) que na masculina (5\%). Posteriormente, outras pesquisas encontraram dados semelhantes, demonstrando a maior vulnerabilidade feminina para o desenvolvimento do transtorno 9 . Algumas explicações para essa maior vulnerabilidade feminina foram levantadas, e a ocorrência de abuso sexual e estupro aumentaria a suscetibilidade para o transtorno. E pelo fato de as mulheres serem as maiores vítimas de violência sexual durante a vida, um número maior de indivíduos do sexo feminino acabaria desenvolvendo o TEPT 29.

Outros fatores relacionados à maior suscetibilidade a TEPT são descritos na literatura. Dentre eles, destaca-se a vulnerabilidade genética, que, como em outros transtornos psiquiátricos, está sujeita à modulação ambiental ${ }^{30}$. A reação diferenciada a situações de estresse, objetivamente mensurada pela secreção de cortisol, já foi relatada ${ }^{31}$. Interessante lembrar que fatores relacionados à hereditariedade quando se trata de transtornos psiquiátricos, além de terem sua expressão modulada pelo ambiente, também podem ser comuns entre si. Por exemplo, as alterações genéticas (expressão de alelos que codificam receptores) que predispõem ao abuso de substâncias também são encontradas em transtornos relacionados como transtorno da conduta e impulsivos ${ }^{32}$. É possível que a genética predisponente à maior responsividade ao estresse e ao abuso de substâncias também torne mais vulnerável o indivíduo exposto a eventos traumáticos e ao desenvolvimento de TEPT. Entretanto, as evidências atuais para tal conclusão ainda são insuficientes.

A relação entre TEPT e cognição e sua evolução para transtornos demenciais ${ }^{33}$ também foi relatada, assim como a eficácia da terapia cognitivo-comportamental no tratamento do TEPT ${ }^{34}$. Outra vertente teórica que vem se tornando muito instigadora nos últimos anos é a relação entre transtornos psiquiátricos e o sistema imune. Diferenças na expressão de interleucinas diante do processo inflamatório foram relacionadas ao TEPT 35 .

Com a ampliação do conceito de evento traumático na quarta edição do DSM, tem sido estudado o aparecimento de sintomas do transtorno em pessoas que fazem parte do cotidiano de hospitais, como familiares de pacientes que estão com a vida ameaçada e não recebem qualquer apoio terapêutico. É o que demonstra trabalho recente que descreve esse transtorno em pais de crianças diagnosticadas com fibrose cística ${ }^{36}$.

Por outro lado, tem havido debate na literatura a respeito da aplicabilidade dos critérios diagnósticos do DSM-IV para comparação entre culturas. O debate possivelmente levará a mudanças na classificação do DSM-V. Áreas que demandam mais pesquisas para que o diagnóstico se torne válido entre as diferentes culturas incluem o papel da interpretação dos sintomas causados pelo trauma e a prevalência de sintomas somáticos, de sonhos angustiantes sobre o evento e de perspectiva reduzida de futuro ${ }^{37}$. É possível que os critérios estabelecidos atualmente sejam mais apropriados para a cultura ocidental. Entre etnias também foram encontradas diferenças de prevalência, com mulheres afro-americanas demonstrando maior frequência de exposição a traumas e TEPT do que americanas de origem não africana ${ }^{38}$. Com o progresso das pesquisas, será possível avaliar a validade desse diagnóstico que exige como critério a exposição a um trauma que ameaça a vida, talvez o único diagnóstico psiquiátrico que tem como critério a presença do fator desencadeador.

Apesar da presença de grau elevado de ansiedade e moderado de depressão, raras voluntárias relataram tratamento. Ao contrário, muitas referiram comprar clandestinamente "calmantes" para conseguir suportar o sofrimento psíquico. Mulheres apresentam um risco significativamente maior comparado com o dos homens para desenvolvimento de transtornos de ansiedade ao longo da vida ${ }^{29}$.

A prisão para as mulheres é uma dupla condenação. Além da perda da liberdade, o encarceramento afeta os núcleos familiares, comunitários e sociais, principalmente por limitar a relação mãe-filho. Muitas vezes, sem justificativa em relação à ausência da mãe, esse filho é exposto ao trauma da separação, que poderá vir a influenciar na perpetuação da violência entre as gerações ${ }^{27}$. As internas relatam ser muito doloroso o cumprimento da pena sem a interação com os filhos devido às escassas ou nenhuma visita ${ }^{27}$.

Considerando que essas mulheres não possuem privacidade, lazer, raramente recebem visitas e estão afastadas de seus filhos, a presença de ansiedade e depressão, principalmente entre as participantes TEPT-positivas, pode ter favorecido o uso de cocaína, calmantes e nicotina, drogas reconhecidamente de consumo aumentado em situações ambientais adversas.

Durante a caracterização sociodemográfica da amostra, surgiu a dificuldade para estabelecer o parâmetro de definição da existência de um companheiro. Muitas mulheres possuíam companheiro antes da prisão, mas haviam sido deixadas desde então ou desconheciam se seriam recebidas ou não pelos companheiros quando saíssem do cárcere. Ao mesmo tempo, o desenvolvimento de laços afetivos e amorosos dentro da prisão, entre as próprias detentas, foi muito relatado. A estruturação das relações interpessoais no ambiente prisional é um tópico singular que deve ser estudado à parte.

Em conclusão, os dados indicam presença de TEPT entre as detentas do presídio, que representa a porta de entrada para o sistema penitenciário do Rio de Janeiro, comparadas às mais altas frequências descritas na literatura para populações expostas à guerra. Os dados também indicaram presença de depressão e maior consumo 
de cocaína entre as detentas TEPT-positivas. A extrapolação desses dados para a população carcerária feminina em geral não pode ser feita dentro das limitações da amostra estudada. Entretanto, dadas as implicações desses resultados para o estabelecimento de medidas políticas e de saúde, espera-se que possam contribuir para a real recuperação de pessoas que estiveram sob a custódia do estado. Embora limitados a uma prisão e a uma centena de participantes, os resultados corroboram os obtidos em outros estudos que apontam no mesmo sentido.

\section{Agradecimentos}

Os autores são particularmente gratos ao Dr. George Christo, pela orientação na aplicação dos instrumentos, e a Beatriz Paulino, pela grande dedicação na coleta, análise e redação do manuscrito até seu precoce e inesperado falecimento em fevereiro de 2010. A ela nossa lembrança carinhosa.

\section{Referências}

1. Bleich A, Siegel B, Garb R, Lerer B. Post-traumatic stress disorder following combat exposure: clinical features and psychopharmacological treatment. Br J Psychiatry. 1986;149:365-9.

2. Kessler RC, Sonnega A, Bromet E, Hughes M, Nelson CB. Posttraumatic stress disorder in the National Comorbidity Survey. Arch Gen Psychiatry. 1995;52(12):1048-60.

3. American Psychiatric Association. Diagnostic and statistical manual of mental disorders. 4th ed. (DSM-IV). Washington: American Psychiatric Association; 1994.

4. Helzer JE, Robins LN, McEvoy L. Posttraumatic stress disorder in the general population. N Engl J Med. 1987;317(26):1630-4.

5. United Nations Office on Drugs and Crime (UNODC). Ninth United Nations Survey of Crime Trends and Operations of Criminal Justice Systems (2003-2004). 2006. Disponível em: http://www.unodc.org/ pdf/research/9th_survey/CTS9ByIndicatorExtract.pdf. Acesso em: 1 maio 2009.

6. Ministério da Justiça. Secretaria Nacional de Segurança Pública - SENASP/Departamento de Pesquisa, Análise da Informação e Formação de Pessoal em Segurança Pública. Análise das ocorrências registradas pelas polícias civis (janeiro de 2004 a dezembro de 2005). 2006. Disponível em: http://www.mj.gov.br/senasp/estatisticas/mapacrime/ Mapacrime2004_2005.pdf. Acesso em: 1 maio 2009.

7. Machado-Vieira R, Gauer GC. Transtorno de estresse pós-traumático e transtorno de humor bipolar. Rev Bras Psiquiatr. 2003;25(Supl 1):55-61.

8. Reed E, Raj A, Falbo G, Caminha F, Decker MR, Kalied DC, et al. The prevalence of violence and relation to depression and illicit drug use among incarcerated women in Recife, Brazil. Int J Law Psych. 2009;32:323-8.

9. Jordan BK, Schlenger WE, Fairbank JA, Caddell JM. Prevalence of psychiatric disorders among incarcerated women. II: Convicted felons entering prison. Arch Gen Psychiatry. 1996;53:513-9.

10. Brady KT, Back SE, Coffey SF. Substance abuse and posttraumatic stress disorder. Curr Dir Psychol Sci. 2004;13(1):206-9.

11. Chilcoat HD, Breslau N. Posttraumatic stress disorder and drug disorders: testing causal pathways. Arch Gen Psychiatry. 1998;55(10):913-7.

12. Christo G, Morris C. Substance-misusers' anxiety and traumatic event prevalence. Drugs Educ Prev Pol. 2004;11(1):35-47.

13. Carvalho ML, Valente JG, Assis SG, Vasconcelos AG. Modelo preditivo do uso de cocaína em prisões do estado do Rio de Janeiro. Rev Saude Publica. 2005;39(5):824-31.

14. Christo G, Silva VA. A Portuguese version of the Christo Inventory for Substance-Misuse Services: a simple outcome evaluation tool. Braz J Med Biol Res. 2002;35(10):1111-7.

15. Christo G. Christo Inventory of Drugs [thesis]. London: King's College; 1996.
16. Spielberger C, Gorsuch RJ, Lushene RE. Inventário de Ansiedade Traço-Estado (IDATE) - Manual de Psicologia Aplicada. Rio Janeiro: Centro de Psicologia Aplicada; 1979.

17. Beck AT, Steer RA. Beck Depression Inventory. San Antonio: Psychological Corporation; 1993.

18. Figueira I, Mendlowicz M. Diagnóstico do transtorno de estresse pós-traumático. Rev Bras Psiquiatr. 2003;25(Supl I):12-6.

19. Gorenstein C, Andrade L, Zuardi AW, editores. Escalas de Avaliação Clínica em Psiquiatria e Psicofarmacologia. São Paulo: Lemos; 2000.

20. Gorenstein C, Andrade L. Validation of a Portuguese version of the Beck Depression Inventory and the State-Trait Anxiety Inventory in Brazilian Subjects. Braz J Med Biol Res. 1996;29(4):453-7.

21. Frinhani FMD, Souza L. Mulheres encarceradas e espaço prisional: uma análise de representações sociais. Psicologia: Teoria e Prática, 2005;7(1):61-79.

22. Freudenberg N. Jails, prisons and the health of urban populations: a review of the impact of the correctional system on community health. J Urban Health. 2001;78(2):214-35.

23. Carvalho ML, Valente JG, Assis SG, Vasconcelos AG. Perfil dos internos no sistema prisional do Rio de Janeiro: especificidades de gênero no processo de exclusão social. Ciênc Saúde Coletiva. 2006;11(2):461-71.

24. Quello SB, Brady KT, Sonne SC. Mood disorders and substance abuse disorders: a complex comorbidity. Sci Pract Perspect. 2005;3(1):13-24.

25. Volkow ND. The reality of comorbidity: depression and drug abuse. Biol Psychiatry. 2004;56(10):714-7.

26. Masho SW, Ahmed G. Age at sexual assault and posttraumatic stress disorder among women: prevalence, correlates, and implications for prevention. J Womens Health (Larchmt). 2007;16(2):262-71.

27. Falbo G, Caminha F, Aguiar F, Albuquerque J, Chacon ML, Miranda S, et al. Incidence of child and adolescent abuse among incarcerated females in the Northeast of Brazil. J Trop Pediatr. 2004;50(5):292-6.

28. Kimerling R, Clum GA, Wolfe J. Relationships among trauma exposure, chronic posttraumatic stress disorder symptoms, and self-reported health in women: replication and extension. J Trauma Stress. 2000;13(1):115-28.

29. Kinrys G, Wygant LE. Transtornos de ansiedade em mulheres: gênero influencia o tratamento? Rev Bras Psiquiatr. 2005;27(Supl 2):S43-50.

30. Koenen KC, Amstadter AB, Nugent NR. Gene-environment interaction in posttraumatic stress disorder: an update. J Trauma Stress. 2009;22(5):416-26.

31. Board R, Dai J. Effects of five parent-and-child risk factors on salivary cortisol levels and symptoms of posttraumatic stress disorder in school-age, critically ill children: pilot study. Heart Lung. 2011;40(3):236-46.

32. Ducci E, Goldman D. Genetic approaches to addiction: genes and alcohol. Addiction. 2008;103(9):1914-28.

33. Cardenas VA, Samuelson K, Lenoci M, Studholme C, Neylan TC, Marmar $\mathrm{CR}$, et al. Changes in brain anatomy during the course of posttraumatic stress disorder. Psychiatry Res. 2011;193(2):93-100.

34. Passarela CM, Mendes DD, Mari JJ. Revisão sistemática para estudar a eficácia de terapia cognitivo-comportamental para crianças e adolescentes abusadas sexualmente com transtorno de estresse pós-traumático. Rev Psiq Clín. 2010;37(2):60-5.

35. Smith AK, Conneely KN, Kilaru V, Mercer KB, Weiss TE, Bradley B, et al. Differential immune system DNA methylation and cytokine regulation in post-traumatic stress disorder. Am J Med Genet B Neuropsychiatr Genet. 2011;156(6):700-8.

36. Cabizuca M, Mendlowicz M, Marques-Portela C, Ragoni C, Coutinho ESF, Souza W, et al. Os pacientes invisíveis: transtorno de estresse pós-traumático em pais de pacientes com fibrose cística. Rev Psiq Clín. 2010;37(1):6-11.

37. Hinton DE, Lewis-Fernández R. The cross-cultural validity of posttraumatic stress disorder: implications for DSM-5. Depress Anxiety. 2011;28(9):783-801.

38. Seng JS, Kohn-Wood LP, McPherson MD, Sperlich M. Disparity in posttraumatic stress disorder diagnosis among African American pregnant women. Arch Womens Ment Health. 2011;14(4):295-306. 